\title{
An Enhanced Optimal Technique For Accurate Detection Of Color Face Images With Different Illuminations
}

\author{
Ms. Meenakshi Shunmugam a ${ }^{\text {, Dr.T.Suresh }}{ }^{\text {b }}$, Dr.M.S.Anbarasi ${ }^{\mathrm{c}}$ and Mr.Manjunath Jayaprakash ${ }^{\text {d, }}$ \\ a Assistant Professor, Department of Electronics and Communication Engineering, R.M.K. Engineering college, Thiruvallur \\ district, Tamilnadu, India. Email: shunmugam.meenakshi@gmail.com \\ b Professor \& Head, Department of Electronics and Communication Engineering, R.M.K. Engineering college, Thiruvallur \\ district, Tamilnadu, India.Email: hod.ece@rmkec.ac.in \\ c Assistant Professor (Sr.Grade), Department of Information Technology, Pondicherry Engineering College. \\ Pondicherry.Email: anbarasims@pec.edu \\ ${ }^{d}$ Senior Engineer - J Ray McDermott Engineering Pvt.Ltd, Chennai Tamilnadu, India. Email: manjunath2k6@gmail.com
}

Article History: Received: 11 January 2021; Accepted: 27 February 2021; Published online: 5 April 2021

\begin{abstract}
Secured authentication system is one of the most challenging tasks focused now-a-days by many researchers and greatly achieved by means of face detection technique. Face image recognition is currently realized by Adaptive singular value decomposition in two-dimensional discrete Fourier domain (ASVDF). The recognition systems ability enhancement is attained for face images recognition by side light influence reduction on a color face image for inadequate light. The prevailing researches does not focuses the following points: No correct output during face recognition process, Face spoofing is not concentrated thereby face recognition may effect in imprecise result, Optimal feature extraction. Optimized Face Recognition System with Illumination and Rotation Consideration (OFRS-IRC) is one of the promising solutions for mitigating all those issues. Various methods are presented for ensuring accurate face recognition. Additive White Gaussian Noise removal technique is utilized for eliminating noise when the image is captured through sensor devices. Illuminate invariant features and locality preserving projection approach is exploited for segmented image recognition. As a final step, Fuzzy neural network is deployed for precise prediction on the basis of locality preserving projection approach results. MATLAB simulation tool is exploited for evaluating this research, where improved performance are attained by proposed method than prevailing methods. The proposed method shows $7.42 \%$ better detection rate than the existing work.
\end{abstract}

Keywords: Face detection, varying illuminations, noise removal, locality preserving, fuzzy neural network, graph segmentation

\section{Introduction}

Generally biometrics, information security, in addition access control and surveillance system predominantly demands face recognition which is regarded as distinguished research areas in pattern recognition as well as computer vision [1]. Apart from this, various applications like content-based image retrieval, video coding, video conferencing, crowd surveillance, besides intelligent human- computer interfaces also requires Automatic face recognition face recognition [2]. The human beingsrecognition can be easily done by a person or friend. Biometric characteristic eases the identification of a person, whereas person identification through computer vision is most difficult task. There is no rigid structure for human faces which adds more complexity. Also, facial changes occurs over a passage of time [3]. Pattern recognition (PR) and artificial intelligence (AI) greatly necessitates automatic face recognition.

The difficulties encompassed with other biometric or ID card/Password based technologies can be avoided by this facial recognition system which may save time and money [4]. The human face is considered as unique verification information in face recognition technology. The facial recognition system is helpful for various operating surroundings from specific home surroundings to most common public places [5]. The Face Recognition System mainly relies man and machine interface technology without any contact. Biometric face detection as well as face verification system offers improved accuracy and consistent security. The face authentication approaches are greatly utilized for "Access Control, Time Attendance System, and Visitor Management System" [6].

Image verification can be done even in very poor lighting [7] which is the primary objective of this research. The proposed system functions efficiently for image recognition or identification though various changes may happen during image lighting. Face is regarded as primary feature for identification and face detection in digital image has its significance in various fields over the last decade [8]. Several image processing techniques are also greatly necessitated by the computer for automation process [9]. Face detection refers to process involved in human faces isolation from their background along with accurate location of their position in an image [10]. A facial recognition system is a computer application which recognizes/substantiates a person from a digital image or a video frame from a video source in an automatic manner. The facial features which are selected from the image are compared with that of facial database [11] which is mainly utilized in security systems besides comparing with other biometrics for instance fingerprint or eye iris recognition systems.

The research work mainly concentrates on developing a system for accurate facial recognition irrespective of diverse illuminations existence in addition to noises in the input images. The implementation of this system is 
done by noise removal and adopting segmentation techniques for facial feature separation which ensures precise recognition. Also recognition rate is achieved precisely through illumination invariant features extraction along with locality preserving features from the input image. At last, fuzzy neural network is presented for assuring the prediction rate.

The research work is organized in the following manner: Section 1 presents a brief introduction about the face image recognition with its requirement. Related works on face recognition techniques is presented in section 2 . Proposed methodology with the block diagram, working procedure and algorithm are explained elaborately in section 3. Section 4 discusses about research work performance analysis depending on the outcomes obtained. Section 5 concludes the research work on the basis of simulation outcome.

\section{RELATED WORKS}

Le et al [12] developed an approach namely Face Relighting As Data Augmentation (FRADA) for estimating 3D morphable model coefficients besides spherical harmonic lighting coefficients. Various parameters such as face normals, face mask, face shading, and face albedo are extracted using this technique. Also new face images under random lighting conditions are obtained by physically-based image formation theory. FRADA is validated by the qualitative outcomes which are more realistic than the traditional face relighting algorithm.

Nehru et al [13] utilized Viola Jones algorithm for human face detection through which automatic identification of human faces is achieved from the specified images regardless of illumination conditions by means of training. Essa et al [14] represented face images by utilizing a combination of local edge gradient information from two dissimilar neighbouring pixel configurations by an illumination invariant face recognition method. Different face images patterns encoding under various lighting conditions are achieved by proposing an oriented local descriptor namely Local Boosted Features (LBF). The local edge response values in different directions along with multi-region histograms from every neighbourhood size are greatly utilized in this research. Long LBFfeature vector for every image is obtained by concatenating these histograms.

Subramanyam et al [15] performed classification on the basis of their illumination and contrast quality for obtaining qualitative four classes. The proper enhancement technique is exploited is chosen for specific class for obtaining best possible image. Yale B database is utilized in this research for experimentation and accuracy rate of $97.14 \%$ is attained by accurate classification of images into appropriate classes.

Roy et al [16] adopted a new methodology termed as local-gravity-face (LG-face) for illumination-invariant and heterogeneous face recognition (HFR). Local Gravitational Force Angle (LGFA) is main notion deployed in LG-face. LGFA corresponds to the gravitational force direction in which center pixel exerts on other pixels contained by local neighbourhood.

Pavlović et al [17] suggested an effective algorithm for illumination invariant face recognition system for developing a visible light face recognition systems in which benefits are taken from both spectra. Infrared facial images is exploited to solve this issue. Thermal IR imagery affords recognition under all lighting environments, comprising comprehensive darkness and closely invariant to variations in illumination.

Ding et al [18] achieved pose-invariant face recognition by proposing a very efficient and accurate pose normalization methodology. Initially, feature extraction in a pose adaptive way can be greatly achieved by 3D facial landmarks projection to every 2D face image. An optimal warp estimation is done on the basis of homography for texture deformation correction affected through pose variations for local patch nearby every landmark. Face recognition with conventional face descriptors is attained by means of reconstructed frontal-view patches.

Dewantara et al [19] presented a new illumination invariant technique namely OptiFuzz for mitigating the illumination effect on face detection along with face recognition issue. The illumination effect for photometricbased human face recognition is elucidated by this optimized fuzzy-based illumination invariant technique. Genetic algorithm is utilized for Fuzzy Inference System rule optimization.

Dhekane et al [20] utilized uniform local binary patterns (ULBP) as well as Legendre moments for illumination as well as expression invariant face recognition technique. Feature representation with improved discriminating power is achieved by this uLBP texture features in addition to Legendre moments. The input images preprocessing is done for extracting normalized face region. The uLBP codes extraction is performed from normalized image for obtaining texture image by which monotonic temperature changes effect is evaded.

Chen et al [21] performed optimization by suggesting a new triplet-loss training process rather than Euclidean loss. There exist two benefits: a) The training tripletsaugmentation can be effortlessly done through freely selecting labelled face images combinations by which overfitting might be evaded; b) The triplet-loss training produces PII features additional discriminative even when training samples possess alike appearance.

Thamizharasi et al [22] exploited 2D Discrete Cosine Transform as well as Contrast Limited Adaptive Histogram Equalization (CLAHE) for designing an illumination invariant face recognition system. The reduced contrast medical images are enhanced using Contrast Limited Adaptive Histogram Equalization. The $75 \%$ to $100 \%$ DCT coefficients are chosen by suggested technique for setting high frequency to zero. On the basis of 
selection percentage, image resizing is done and inversed DCT is utilized. The contrast adjustment is attained by CLAHE and computational complexity is reduced by resized images.

\section{ILLUMINATION CNCERNED FACE IMAGE RECOGNITION}

This research exploits various methods for ensuring accurate face recognition. Additive White Gaussian Noise removal technique is utilized for eliminating noise when the image is captured through sensor devices. Graph based segmentation is employed next for properly segmenting the facial segment of the images for precise face recognition. Illuminate invariant features and locality preserving projection approach is exploited for segmented image recognition. As a final step, Fuzzy neural network is deployed for precise prediction on the basis of locality preserving projection approach results. The research work processing flow is depicted in figure 1.
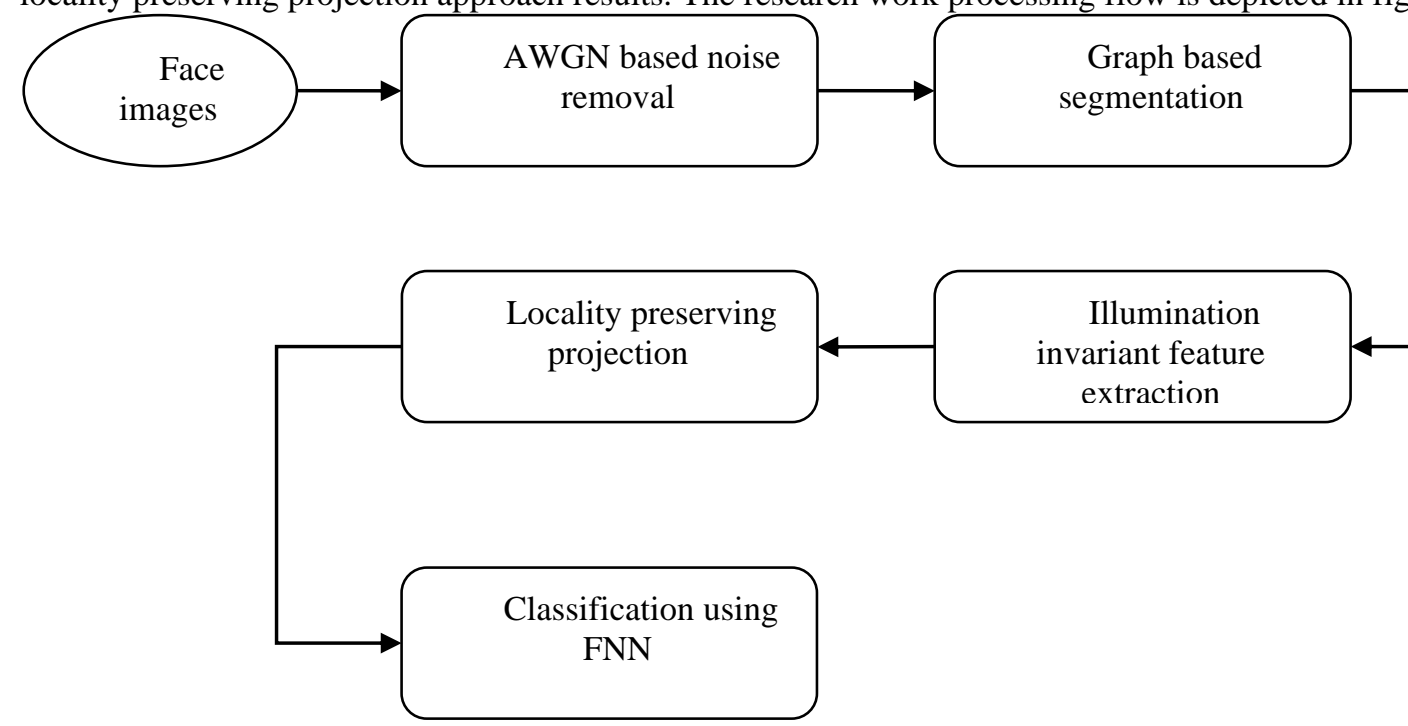

Figure 1. Proposed research work processing flow

\subsection{ADDITIVE WHITE GAUSSIAN NOISE REMOVAL}

Many random processes effect might arise in nature which can be alleviated by Additive white Gaussian noise (AWGN) which is regarded as simple noise model employed in information theory. Specific characteristics are represented by modifiers:

- Additive as it is added to any noise which could be intrinsic to information system.

- Similarly, like colour white notion, this method possesses constant power across frequency band for information system, as white has uniform emissions at all frequencies in visible spectrum.

- Gaussian, since it has normal distribution in time domain with an average time domain value of zero.

From various natural sources (atoms thermal vibrations in conductors (referred as thermal noise or JohnsonNyquist noise), shot noise), black-body radiation from earth besides other warm objects, in addition from celestial sources (Sun), Wideband noise emerges. The central limit theorem of probability theory represents summation tendency of numerous random processes holds distribution, namely Gaussian or Normal.

AWGN is a frequently-used channel model, where only impairment to communication is a wideband or white noise linear addition with a constant spectral density (expressed as watts per hertz of bandwidth) along with amplitude Gaussian distribution. The interference, frequency selectivity, fading, dispersion or nonlinearity is not considered. Nevertheless, the mathematical models generated by this approach proved to be simple besides tractable, which are beneficial for acquiring insight into underlying framework behaviour prior to the consideration of these other phenomena. The AWGN channel proves its significance in several satellite and deep space communication links. Due to the multipath, terrain blocking, interference, etc., this model is inadequate for most terrestrial links. But still, AWGN has widely utilized in terrestrial path modelling, in order to perform the simulation of channel background noise under study, apart from multipath, terrain blocking, interference, ground clutter as well as self-interference that modern radio systems come across in terrestrial operation.

\subsection{GRAPH BASED SEGMENTATION}

From the computer perspective, the image segmentation process confers digital image partitioning, where image gets divided into numerous segments (super pixels/sets of pixels). Simplification or/and modification of the image representation are the core intentions of this process, in order to make the image more suggestive and make ease of analysis. In a 2D or 3D image, the Face Segmentation detects the boundaries either automatically or semi-automatically. However, the high variability in face images considered being the biggest challenge of face segmentation. Primarily, there are significant modes of variation within the human anatomy. Also, numerous modalities, such as MRI, CT, microscopy, Endoscopy, PET, OCT, SPECT, X-ray, etc., have utilized to create face images. Subsequently, for attaining additional diagnostic acumen, the segmentation result helps. 
According to the extracted boundary information, applications such as automatic measurement of organs, cell counting, or simulations have carried out feasibly.

Throughout this study, face image segmentation has executed through adapting the graph based segmentation. During this process, the fine level details of the face images have highly focused to enhance the compression result. In addition, efficient graph-based image segmentation has further involved to perform the clustering in feature space. Here, this framework directly operates over the data points, deprived of filtering step as an initial process, and utilizes a variation across single linkage clustering. Adaptive thresholding has regarded as the vital factor for the success of this approach. Conventional single linkage clustering has performed by initially generating a data points minimum spanning tree with the help of Kruskal's algorithm which enables the removal of edges, whose length is larger than a provided hard threshold. In segmentation process, associated components turnout to be the clusters. The requirement for a hard threshold has eradicated by this model, besides switched it with a data-dependent term. Particularly, consider $\mathrm{G}=(\mathrm{V}, \mathrm{E})$ as a (fully connected) graph accompanying $\mathrm{m}$ edges as well as $\mathrm{n}$ vertices, where each vertex is a pixel, $\mathrm{x}$, denoted in feature space. Then, final segmentation corresponds to $\mathrm{S}=\left(\mathrm{C}_{1}, \ldots, \mathrm{Cr}\right)$ where $\mathrm{Ci}$ is data points cluster. Algorithm for this process can be expressed as,

1. Classify $\mathrm{E}=\left(\mathrm{e}_{1}, \ldots, \mathrm{e}_{\mathrm{m}}\right)$ such that $\left|\mathrm{e}_{\mathrm{t}}\right| \leq\left|\mathrm{e}_{\mathrm{t}^{\prime}}\right| \forall \mathrm{t}<\mathrm{t}^{\prime}$

2. Let $S^{0}=\left(\left\{x_{1}\right\}, \ldots,\left\{x_{n}\right\}\right)$, in other words every initial cluster comprises precisely one vertex.

3. For $\mathrm{t}=1, \ldots, \mathrm{m}$

(a) Consider $\mathrm{x}_{\mathrm{i}}$ and $\mathrm{x}_{\mathrm{j}}$ as vertices connected by $\mathrm{e}_{\mathrm{t}}$.

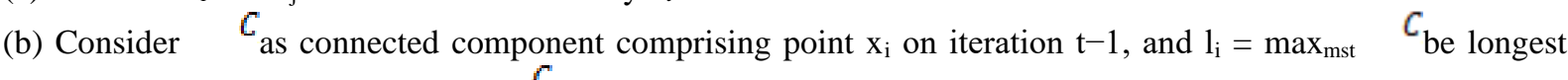
edge in minimum spanning tree of ${ }^{C}$. Likewise for $l_{\mathrm{j}}$.

(c) Combine $\quad C_{\text {and }} C_{\text {if }}$

Here, $\mathrm{k}$ notates constant.

$$
\left|\mathrm{e}_{\mathrm{t}}\right|<\min \left\{1_{\mathrm{i}}+\frac{k}{\left|c_{x_{i}}^{\mathrm{i}-1}\right|}, l_{j}+\frac{}{\sigma}\right.
$$

4. $S=S^{m}$

The segmented parts of the face images can be obtained through applying this algorithm.

\subsection{ILLUMINATION INVARIANT FEATURE EXTRACTION}

Let faces to be Lambertian surfaces, provided face image I under specific illumination criteria and specified as reflectance and illumination product . It can be expressed as,

$\mathrm{I}(\mathrm{x}, \mathrm{y})=\mathrm{L}(\mathrm{x}, \mathrm{y}) \mathrm{R}(\mathrm{x}, \mathrm{y})$

In which, at point $(x, y)$, illuminance and reflectance is represented by $\mathrm{L}(\mathrm{x}, \mathrm{y})$ and $\mathrm{R}(\mathrm{x}, \mathrm{y})$, correspondingly. Here $\mathrm{R}(\mathrm{x}, \mathrm{y})$ is determined, since it is known as image illumination invariant part . Consider, if the illumination lies in image low-frequency part despite the fact that reflectance, then it is considered to be image high-frequency part . As per the physiological record, the retina cells' response to image intensity on the retina has proved to be a nonlinear function, besides it might be considered every pixel's intensity logarithm. The logarithm reduces bright pixel values range and multiplies dark pixel values range when it gets applied over an image. Following equation expresses logarithm of I,

$\mathrm{I}^{\prime}=\log (\mathrm{I})=\log (\mathrm{L})=\log (\mathrm{R})=\mathrm{L}^{\prime}+\mathrm{R}^{\prime}$

In which, reflectance and illumination logarithms is represented as R' and L', correspondingly. Consider if illumination lies in image low frequency part I, then image illumination invariant information can be attained through the application of 2D high-pass filter on I'. The illumination invariant has considered as the high-pass filter output, which can get utilized for recognition step, e.g. by PCA/LDA. Due to image illumination invariant part requirement, the application of 2D low-pass filter over input image logarithm helps to derive L', and subtracts the output from I'. Consequently, an estimation of R' can be the result image J. Being the standard components in image processing, 17 individual high-pass and 31 low-pass 2D filters have exploited in two structures to acquire the illumination invariants. The maximum, Median, Bilateral in addition Wiener filters have proved to be efficient in obtaining the optimal results.

During the experiments carried out with support vector machine (SVM), k-nearest neighbors (kNN) as well as fuzzy $\mathrm{kNN}$ classifiers, maximum filter proves its efficiency to obtain optimal outcomes in various face recognition. As a nonlinear 2D low-pass filter, the maximum filter has utilized in image processing as regards the removal of negative outlier noise. For every output image pixel, B, intensity value refers to maximum value in an $\mathrm{m} \times$ nneighborhood in input $\mathrm{A}$,

$\mathrm{B}(\mathrm{x}, \mathrm{y})=\max \mathrm{A}(\mathrm{i}, \mathrm{j})$ and $(\mathrm{i}, \mathrm{j}) \quad\{\mathrm{m} \times \mathrm{n}$ neighborhood of $(\mathrm{x}, \mathrm{y})\}$

Following steps define the proposed approach for the illumination invariants extraction

a) Detection and cropping of face in input image, as well as resizing into $\mathrm{W}_{1} \times \mathrm{W}_{2}$ image $\mathrm{I}$ as regards the reduction of computation cost.

b) Image logarithm estimation of I, I'.

c) Computation of low frequency part of I', $\mathrm{K}$, by executing an $\mathrm{m} \times \mathrm{n}$ maximum filter on I'. 
d) Subtracting K from I' to generate improved image $\mathrm{J}$.

$\mathrm{J}=\mathrm{I}^{\prime}-\mathrm{K} \quad \mathrm{R}^{\prime}$

\subsection{FACE RECOGNITION USING LOCALITY PRESERVING FEATURES}

For addressing the present-day requirements regarding the identification and validation of identity claims, face recognition strategy turns out to be captivating solution. There are numerous face recognition frameworks have emerged that accompany various levels of achievements through the enhancements made on feature extraction approaches along with dimensionality reduction methods in pattern recognition application. Recently, Locality preserving projection (LPP) has took place to carry out unsupervised linear dimensionality reduction, which tend to conserve face image space local structure, since it has considered being highly important than global structure which has preserved via principal component analysis (PCA) as well as linear discriminant analysis (LDA).

Being linear projective maps, Locality Preserving Projections (LPP) efficiently preserves data set neighbourhoodstructure through resolving a variation problem. At the time of high-dimensional data lies over a low dimension manifold embedded in data space, LPP denotes nonlinear Laplacian Eigen maps linear approximation in order to approximate various Laplace-Beltrami operator Eigen functions. Instead of data global structure preservation like PCA and LDA, it tends for data local structure preservation. Besides, as an unsupervised algorithm, LPP executes a linear transformation. Through implementing the construction of adjacency graph that expresses data local nearness, LPP models the manifold structure, which makes face recognition extensively favourable than nonlinear local structure preserving. The reason is that it considerably reduces the computational expense, beside it has designated in training points as well as Iso maps as well as Laplacian Eigen maps. Consider $\mathrm{x}_{\mathrm{i}}, \mathrm{i}=1,2, \ldots, \mathrm{n}$, as training patterns of $\mathrm{m}$ classes, $\mathrm{X}=\left[\mathrm{x}_{1}, \mathrm{x}_{2}, \ldots, \mathrm{x}_{\mathrm{n}}\right]$ as data matrix; $1\left(x_{i}\right)$ as label of $x_{i}$, then $1\left(x_{i}\right)=k$ implies that $x_{i}$ belongs to class $k$. In original data space, LPP forces neighbouring points to get mapped into closely projected data as regards data's intrinsic geometry preservation . The algorithm initiates by assigning a similarity matrix $\mathrm{W}$, in accordance with (weighted) $\mathrm{k}$ nearest neighbours graph, whose entry $\mathrm{W}_{\mathrm{ij}}$ signifies edge amid training images (graph nodes) $\mathrm{x}_{\mathrm{i}}$ and $\mathrm{x}_{\mathrm{j}}$. Gaussian type weights of form

$W_{i j}=e^{-\frac{\| x_{i}-4}{{ }^{i}}}$ have Jective function has carried out in accordance with matrix $\mathrm{W}$, in which projected data points locality has applied through penalizing points those which are mapped far apart. Basically, this methodology diminishes for obtaining minimum Eigen value solution to generalized Eigen value problem.

\section{ALGORITHM}

Locality Preserving Projection (LPP) has known as linear approximation method derived from nonlinear Laplacian Eigen map [3]. LPP algorithm includes the following procedures

1) Adjacency graph construction: Consider $G$ as a graph with $m$ nodes and an edge amid nodes $I$ and $j$, if $x_{i}$ and $\mathrm{x}_{\mathrm{j}}$ are close. It includes two variations:

(a) $\varepsilon$-neighbourhoods: Nodes $i$ and $j$ are connected through an edge if $\left\|x_{i}-x_{j}\right\|^{2}$, in which norm is usual Euclidean norm in $\mathrm{R}^{\mathrm{n}}$.

(b) $\mathrm{k}$ nearest neighbors: Nodes $\mathrm{i}$ and $\mathrm{j}$ are connected by an edge if iis amid $\mathrm{k}$ nearest neighbors of $\mathrm{j}$ or $\mathrm{j}$ is amid $\mathrm{k}$ nearest neighbors of $\mathrm{i}$.

2) Selection of weights: $\mathrm{W}$ is a sparse symmetric $\mathrm{m}$ m matrix with $\mathrm{W}_{\mathrm{ij}}$ that includes edge joining vertices weight iand $\mathrm{j}$, and 0 if there is no such edge. There are two variations for weighting edges as follows

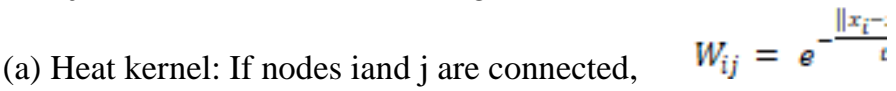

(b) Simple-minded: $\mathrm{W}_{\mathrm{ij}}=1$, if and only if vertices iand $\mathrm{j}$ are connected through an edge.

3) Eigen maps: Calculate eigenvectors besides Eigen values for generalized eigenvector problem:

$\mathrm{XLX}^{\mathrm{T}} \mathrm{a}=\lambda \mathrm{XDX}^{\mathrm{T}} \mathrm{a}$

Here, a diagonal matrix has notated by $\mathrm{D}$, whose entries are column (or row, since $\mathrm{W}$ is symmetric) sums of W, Laplacian matrix has formulated as $\quad D_{\mathrm{ii}}=\Sigma_{j} \quad W_{j \mathrm{i}}, L=D-$, and $\mathrm{x}_{\mathrm{i}} \mathrm{denotes} \mathrm{i}^{\text {th }}$ column of matrix X. Consider column vectors $\mathrm{a}_{0}, \ldots, \mathrm{a}_{\mathrm{l}-1}$ as equation (7) solutions, ordered on the basis of their eigen values, $\lambda_{0}<\ldots . \lambda_{1-1}$. Hence, embedding can be carried out as specified

$\mathrm{x}_{\mathrm{i}} \square \mathrm{y}_{\mathrm{l}}=\mathrm{A}^{\mathrm{T}} \mathrm{x}_{\mathrm{i}}, \mathrm{A}=\left(\mathrm{a}_{0}, \mathrm{a}_{1}, \ldots, \mathrm{a}_{\mathrm{l}-1}\right)$

In which, 1 -dimensional vector has signified by $\mathrm{y}_{\mathrm{i}}$, and $\mathrm{n} 1$ matrix has denoted by $\mathrm{A}$.

\subsection{CLASSIFICATION USING FUZZY NEURAL NETWORK}

For the classification approach, the extraction of feature sets has utilized. MR brain image has classified through Fuzzy Neural Network (FNN), whose outcomes have categorized as Alzheimer's disease, Mild Alzheimer's disease and Huntington's disease. Considering performance metrics, such as accuracy, sensitivity and specificity, classification efficiency system has been evaluated.

The implementation of FNN has carried out in middle of two classes. In a same way, each class of FNN has grouped. In supplementary classes, fuzzy memberships have produced by the additional classes that enable the 
selection of highest value winner in the final output node. In the input, the prototype feature vectors. Here, the exemplar data comprises two classes words, there are two individual labels in $\quad t$. Consequently, $K$ : class groups of hidden nodes have utilized here, where each one of the nodes represents Gaussian function on the basis of exemplar feature vector which includes the connected label. In a class group, every Gaussian relies on dissimilar center, but with similar label. For Class 1, the initial hidden nodes group has considered. In such circumstance, there is a chance that of feature vectors in Class can be a large number (here considered as $p=$ ). Because of this reason, feature vectors those which are close to distinctive feature vector and possessing similar label has removed. This process significantly reduces number of centers, consequently Gaussians (nodes) signify each Class . The Gaussian Fuzzy Set Membership Function (FSMF) centered on ${ }^{2}$ provides fuzzy truth that input vector is in identical class as ${ }^{2}$. Being a function, the "Gaussian FSMF is formulated as follows,

$x \rightarrow g\left(x ; x^{(q)}\right)=\exp \exp \left\{-\frac{\left\|x-x^{(q)}\right\|^{2}}{2 \sigma^{2}}\right\}$

Here, a one-half of average distance that belonging to overall exemplar pairs has denoted by

Here, from the corresponding Gaussian nodes, the overall fuzzy facts for Gaussians centers in Class 1 has given into the Class 1 fuzzy maximize node truths that enacts as a fuzzy OR node in selection process of representative center as well as fuzzy truth that involved in certain ${ }^{2}$ for Class 1 . Final output maximizer node has been provided with this maximum fuzzy truth for in Class 1 as Class 1 representative. Besides, final output maximizer node obtains Class 2 representative (maximum fuzzy truth) that involved in Class 2 besides determines maximum among these fuzzy truth. Ultimetely, class that sent is winner. Hence, input involved in winning class decided with winning Gaussian center vector label.

\section{Algorithm for fuzzy neural network classification}

Being the high-level algorithm, it has considered as nearly a well-defined, as presented in the following steps. It is simple to get processed. For training purpose, expending computation time doesn't necessitated by training as well as learning exist in exemplar feature vector besides corresponding labels. The following steps define the pseudo code algorithm.

Step 1: Obtain the data file (the number of features , the amount of feature vectors, the dimension of the labels, the number of classes, the entire feature vectors and the entire labels).

Step 2: Determine least distance $\quad D_{s}$ among the overall feature vector pairs

Put $\sigma=D_{\min } / 2$

Put $G=Q / /$ starting no. of Gaussian centers

Step 3: Determine two exemplar vectors of min. distance with indices and .

If $d<\left(\frac{1}{2}\right) D_{\min } / /$ if vector are close

If label [k1] = label[k2] // have same label

Eliminate Gaussian center $k 2$

$G=G-1 / /$ reduce no. of Gaussians

Goto step2

Step 4: Feed subsequent unknown $\mathrm{x}$ to SFNN to be classified

For $k=1$ to $G$ do

Compute $g[k]=\exp \left\{-\left\|x-x^{(k)}\right\|^{2} /\left(2 \sigma^{2}\right)\right\}$

Find maximum $g\left[k^{*}\right]$,ober $k=1_{, \ldots,}, G$

Output $x_{\text {s }}$ label $\left[k^{*}\right] / /$ habel $\left[k^{*}\right]$ is class of $x$

Step 5: If all inputs for classifying are completed, stop Else, go to Step 3

4. EXPERIMENTAL RESULTS

During the experiments, the proposed Optimized Face Recognition System with Illumination and Rotation Consideration (OFRS-IRC) has compared with prevailing method, namely Adaptive singular value decomposition in two-dimensional discrete Fourier domain (ASVDF) by considering the metrics, like false acceptance rate, genuine acceptance rate false rejection rate as well as accuracy, concerning the performance evaluation.

FALSE ACCEPT RATE (FAR):

$\mathrm{FAR}=$ Total false Acceptance /Total false Attempts 
FAR refers to an impostor probability, who has recognized as a genuine individual. To be precise, it has estimated as the ratio between the number of falsely accepted people (i.e. accepting false people), among overall people enrolled for a predetermined threshold in a biometric authentication system.

\section{FALSE REJECT RATE (FRR)}

FRR refers to "the probability of a genuine individual being rejected as an impostor". In a biometric authentication system, it has calculated as the ratio between the number of people falsely rejected (genuine people are rejected) among overall enrolled people for a predefined threshold.

FRR= Total False Rejection/ Total True Attempts

\subsection{DETECTION RATE}

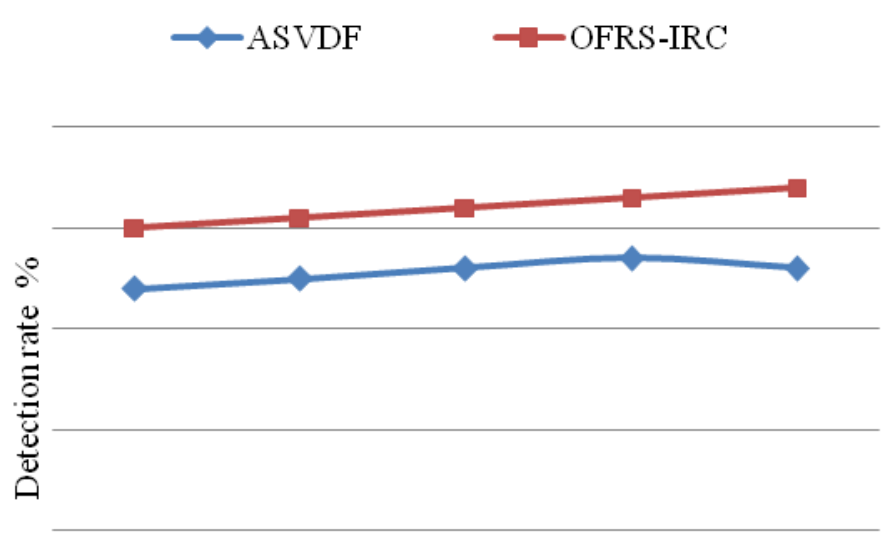

FAR \%

Figure 2. Detection Rate comparison

The detection rate obtained by proposed OFRS-IRC method and prevailing ASVDF method for the face recognition system compared is shown in figure 2, in which $\mathrm{X}$-axis represents the false acceptance rate, in addition Y-axis stands for detection rate. During the experiments, introduced the method, namely Locality Preserving Projections for the process of face recognition that considerably enhances the detection rate. The outcomes demonstrate the proficiency of the proposed OFRS-IRC to accomplish the optimal detection, which is $7.42 \%$ better than other prevailing methodologies.

\subsection{FALSE ACCEPT RATE (FAR) VS FALSE REJECT RATE (FRR)}




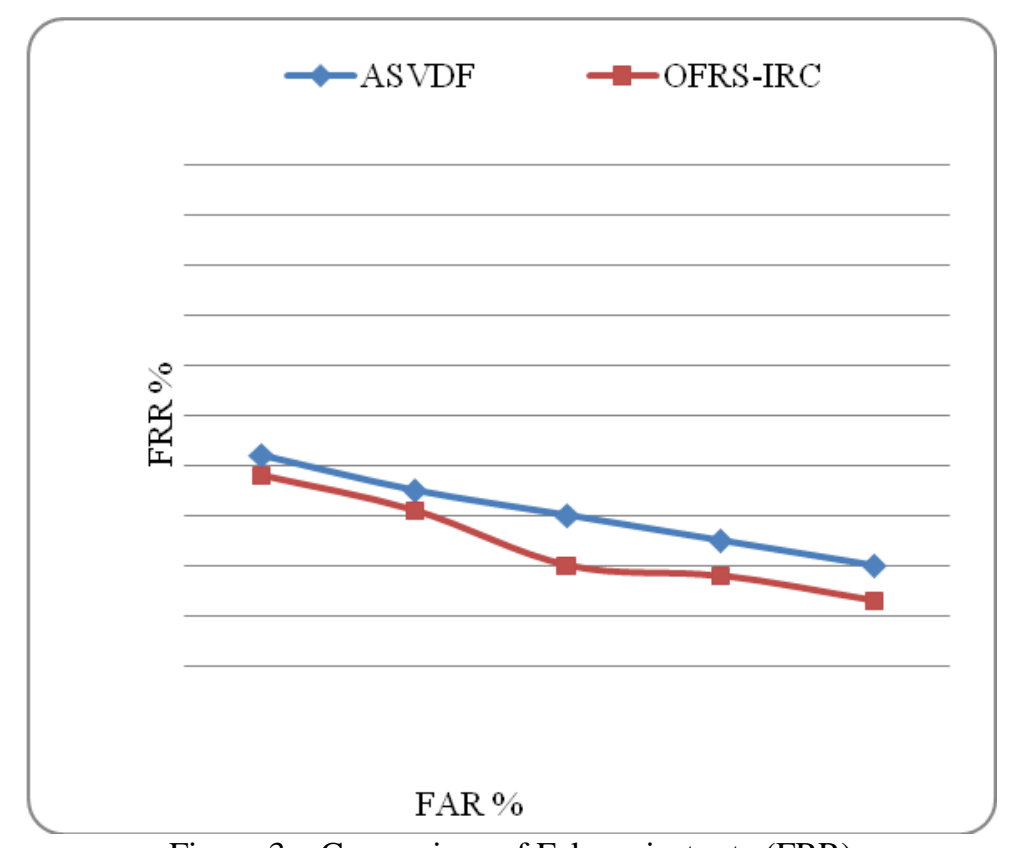

Figure 3. Comparison of False reject rate (FRR)

Figure 3 false rejection rate obtained by proposed OFRS-IRC method and prevailing ASVDF method are compared, $\mathrm{x}$ axis represents false acceptance rate, in addition $\mathrm{y}$ axis stands for false rejection rate. Here, introduced the Locality Preserving Projections that preserves the locality structures for efficient face matching, through which the face recognition has optimized, thereby obtained high safety. Ultimately, the outcomes depict the efficiency of the proposed OFRS-IRC method that exceeds the performance of other prevailing methodologies. From the graphical comparison it is confirmed that the proposed and existing methods false rejection rate reduces linearly as the false acceptance rate increases. The numerical analysis proved proposed OFRS-IRC shows $21.05 \%$ lesser false rejection rate than the existing ASVDF.

\subsection{GENUINE ACCEPTANCE RATE:}

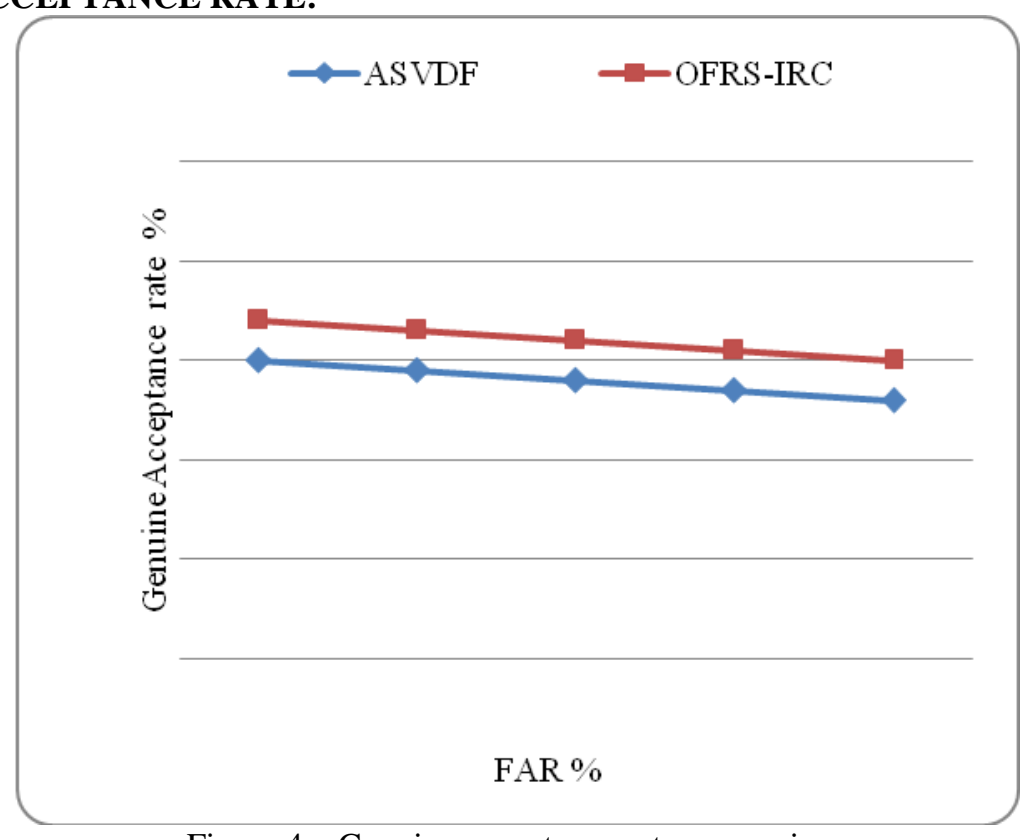

Figure 4. Genuine acceptance rate comparison

In Figure 4, the proposed OFRS-IRC method has compared with prevailing ASVDF method on the basis of genuine acceptance rate. In the figure, the false acceptance rate lies on $\mathrm{X}$-axis, and $\mathrm{Y}$-axis stands for genuine acceptance rate. Being the fraction of genuine scores, the GAR (1-FRR) surpasses the threshold. Besides, the ROC curves represents that the level of performance gain is comparatively great than other prevailing strategies. From the simulation analysis, it is confirmed that the proposed method OFRS-IRC shows $5.12 \%$ increased genuine acceptance rate than the existing ASVDF method. From the graphical analysis it is proved that the genuine acceptance rate reduces linearly as the False acceptance ratio increases.

\section{CONCLUSION}


During this research, several approaches have been presented to guarantee accurate face recognition. Further, Additive White Gaussian Noise removal method takes place to capture the images using sensor devices, which ensures the accuracy of face recognition. In addition, the assurance for accuracy in face recognition has made through presenting the segmentation on the basis of noise removal Graph, as it has the ability to segment the portion of face appropriately from the images. Subsequently, for recognizing this segmented image, the illuminate invariant features and locality preserving projection technique have further involved. Eventually, in accordance with the result derived by locality preserving projection technique, the accurate prediction has made through the fuzzy neural network. Through the evaluation carried out under the simulation environment of MATLAB, the approach proposed in this study delivers the optimal performance, which is superior to other prevailing methods.

\section{References}

1. Masi, I., Wu, Y., Hassner, T., \& Natarajan, P. (2018, October). Deep face recognition: A survey. In 2018 31st SIBGRAPI conference on graphics, patterns and images (SIBGRAPI) (pp. 471-478). IEEE.

2. Zhou, W., Li, H., \& Tian, Q. (2017). Recent advance in content-based image retrieval: A literature survey. arXiv preprint arXiv:1706.06064.

3. Agudo, A., \& Moreno-Noguer, F. (2018). A scalable, efficient, and accurate solution to non-rigid structure from motion. Computer Vision and Image Understanding, 167, 121-133.

4. Gao, J., Rong, Y., Tian, X., \& Yao, Y. O. (2020). Save Time or Save Face? The Stage Fright Effect in the Adoption of Facial Recognition Payment Technology. The Stage Fright Effect in the Adoption of Facial Recognition Payment Technology (August 6, 2020).

5. Hassan, M. M., Uddin, M. Z., Mohamed, A., \&Almogren, A. (2018). A robust human activity recognition system using smartphone sensors and deep learning. Future Generation Computer Systems, 81, 307-313.

6. Ganong, R., Waugh, D. C., Dolejs, J., Wysocki, T., \&Studholme, C. (2019). U.S. Patent No. 10,169,646. Washington, DC: U.S. Patent and Trademark Office.

7. Kedzierski, M., \&Wierzbicki, D. (2016). Methodology of improvement of radiometric quality of images acquired from low altitudes. Measurement, 92, 70-78.

8. Yoganand, A. V., Kavida, A. C., \& Devi, D. R. (2020). Pose and occlusion invariant face recognition system for video surveillance using extensive feature set. International Journal of Biomedical Engineering and Technology, 33(3), 222-239.

9. Sahu, D., \&Dewangan, C. (2017). Identification and classification of mango fruits using image processing. Int. J. Sci. Res. Comput. Sci. Eng. Inf. Technol, 2(2), 203-210.

10. Yusuf, A. A., Mohamad, F. S., \&Sufyanu, Z. (2017). Human face detection using skin color segmentation and watershed algorithm. American Journal of Artificial Intelligence, 1(1), 29-35.

11. Anusha, P., Prasad, K. L., Kumar, G. R., Lydia, E. L., \& Parvathy, V. S. (2020). FACIAL DETECTION IMPLEMENTATION USING PRINCIPAL COMPONENT ANALYSIS (PCA). Journal of Critical Reviews, 7(10), 1863-1872.

12. A Novel Enhanced Gray Scale Adapative Method for Prediction of Breast Cancer, C.Selvi, Dr.M.Suganthi in journal of Medical Systems (2018)

13. Le, H., \&Kakadiaris, I. (2019, January). Illumination-invariant face recognition with deep relit face images. In 2019 IEEE Winter Conference on Applications of Computer Vision (WACV) (pp. 2146-2155). IEEE.

14. Nehru, M., \&Padmavathi, S. (2017, January). Illumination invariant face detection using viola jones algorithm. In 2017 4th International Conference on Advanced Computing and Communication Systems (ICACCS) (pp. 1-4). IEEE.

15. Essa, A., \&Asari, V. K. (2017). Local boosted features for illumination invariant face recognition. Electronic Imaging, 2017(10), 70-73.

16. Subramanyam, B., Joshi, P., Meena, M. K., \& Prakash, S. (2016). Quality based classification of images for illumination invariant face recognition. In 2016 IEEE International Conference on Identity, Security and Behavior Analysis (ISBA) (pp. 1-6). IEEE.

17. Roy, H., \&Bhattacharjee, D. (2016). Local-gravity-face (LG-face) for illumination-invariant and heterogeneous face recognition. IEEE Transactions on Information Forensics and Security, 11(7), 14121424.

18. Kumar, A., Kumar, P., Srivastava, A., Kumar, V., Vengatesan, K., \& Singhal, A. (2020). Comparative Analysis of Data Mining Techniques to Predict Heart Disease for Diabetic Patients. In International Conference on Advances in Computing and Data Sciences (pp. 507-518).

19. Pavlović, M., Stojanović, B., Petrović, R., \&Stanković, S. (2018, November). Fusion of visual and thermal imagery for illumination invariant face recognition system. In 2018 14th Symposium on Neural Networks and Applications (NEUREL) (pp. 1-5). IEEE. 
20. Ding, C., \& Tao, D. (2017). Pose-invariant face recognition with homography-based normalization. Pattern Recognition, 66, 144-152.

21. Dewantara, B. S. B., \& Miura, J. (2016). OptiFuzz: a robust illumination invariant face recognition system and its implementation. Machine Vision and Applications, 27(6), 877-891.

22. Dhekane, M., Seal, A., \& Khanna, P. (2017). Illumination and expression invariant face recognition. International Journal of Pattern Recognition and Artificial Intelligence, 31(12), 1756018.

23. Chen, X., Lan, X., Liang, G., Liu, J., \& Zheng, N. (2017). Pose-and-illumination-invariant face representation via a triplet-loss trained deep reconstruction model. Multimedia Tools and Applications, 76(21), 22043-22058.

24. Thamizharasi, A., \&Jayasudha, J. (2016). An illumination invariant face recognition by selection of dct coefficients. International Journal of Image Processing (IJIP), 10(1), 14.

25. Mallikalava, V.Yuvaraj, S.,Vengatesan, K.,Kumar, A.Punjabi, S.Samee, S.Theft vehicle detection using image processing integrated digital signature based ECU,Proceedings of the 3rd International Conference on Smart Systems and Inventive Technology, ICSSIT 2020August 2020, Article number 9214174, Pages 913-918,

\section{AUTHOR DETAILS}

1.Ms.MeenakshiShunmugam, M.E., is Assistant Professor, Department of Electronics and Communication Engineering, R.M.K. Engineering college.

Email: shunmugam.meenakshi@gmail.com

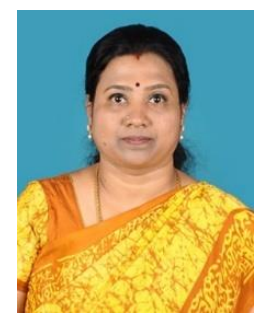

She received her B.E degree in ECE from ACCET., Madurai Kamaraj University and M.E in Applied Electronics fromRMKEC,Anna University. She has been into the teaching profession for 7.2 years . She has 2.9 years of Research experience and industrial experience of one year. Her areas of interest include, Image Processing, Communication Engineering and Control systems. She has published 3 papers in International journals and conferences.

2.Dr.T.Suresh, Professor \& Head, Department of Electronics and Communication Engineering, R.M.K. Engineering college, Thiruvallur district, Tamilnadu, India.

Email: $\underline{\text { hod.ece@rmkec.ac.in }}$

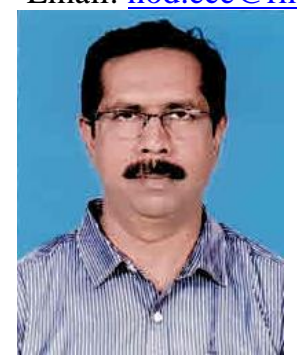

Dr. T. Suresh, M.E., Ph.D, is Professor \& Head, Department of Electronics and Communication Engineering, R.M.K. Engineering college. He obtained his B.E degree in ECE from Madras University, Chennai, M.E., in Microwave and Optical Engineering from Madurai Kamaraj University and Ph.D in VLSI at Anna University. He has been in the teaching profession for the past 26 years. He has 2 years of industrial experience. His areas of interest include, Image Processing, 5G technology, VLSI Design and Embedded system. He is a Life member of ISTE and member of IEEE. He has published more than 30 papers in International journals and conferences.

3.Dr. M.S.Anbarasireceived the B.Tech(Computer Science and Technology) degree from Bangalore University, Bangalore M.E( Software Engineering) and Ph.D. degrees in Data Mining from CEG Campus , Anna University Chennai. She is currently working as Assistant Professor (Sr.Grade) in the Department of Information Technology at Pondicherry Engineering College.

Email: anbarasims@pec.edu 


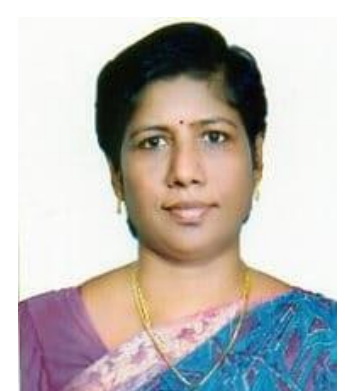

Previously, she served as HOD in VelTechEngineeing College, Chennai then as Research Associate and later as Lecturer in Department of Computer Science and Engineering in College of Engineering, Guindy Campus, Anna University Chennai.

She has published over 44 articles in Springer, INDER SCIENCE, Scopus Indexed, peer reviewed journals and 23 articles in International conferences like IEEE. Her research interests include Data Mining, Big Data Analytics and Cloud Computing.

4.Mr.Manjunath Jayaprakash, Senior Engineer - J Ray McDermott Engineering Pvt.Ltd, Chennai Tamilnadu, India. Email: manjunath2k6@gmail.com

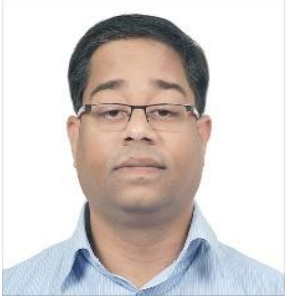

Manjunath Jayaprakash is currently associated with Oil and Gas Engineering, Procurement and Construction company (J Ray McDermott Engineering Pvt.Ltd, Chennai Tamilnadu) as Senior Engineer. He is B.E degree holder in Mechanical Engineering and graduated from Visvesvaraya Technological University in the year 2005. He has 15 years of experience in the field of both onshore and offshore oil and gas engineering design and holds various international work experience around the globe viz., USA, South Korea, Australia, Nigeria, Japan, Qatar. 